\title{
MODELLING OF RED AND WHITE MUSCLE FIBRES IN FORCE GENERATION
}

\author{
Igors Tipans, Janis Viba, Antons Esaulenko \\ Riga Technical University, Latvia \\ igors.tipans@rtu.lv; janis.viba@rtu.lv
}

\begin{abstract}
Force development mechanisms in white and red muscle fibres are investigated. Both types of fibres play different roles in muscular force generation, expressed in force onset velocity and duration. The purpose of the work is to find the key parameters in the model imitating differences in onset velocity for both types of muscle fibre cells, which further would allow to model the propagation of excitation wave. The model allowed to estimate the effect of differences in capacitances of cell membranes of red and white muscle cells to depolarization velocity. With the model we were able to determine the conditions of appearance of low amplitude oscillations interrupting the force generation. Investigation of different behaviour of red and white muscle fibres is needed to understand reaction mechanisms of living objects to sudden external situation changes, such as obstacles or attacks. Similar ability to have two types of force production - slow sustained, and fast of short duration - is often required for robotic devices
\end{abstract}

Keywords: red and white muscle fibres, muscular force generation, modelling.

\section{Introduction}

Red and white muscle fibres are characteristic for cross-striated muscles taking part in force generation in different movements and other muscular activities. White muscular fibres have a typical fast action needed to develop large force in a short time interval, whereas red muscular fibres are typically slow motion fibres able to generate force for a long duration, e.g., to hold a given position of a body [1]. Existence of two different patterns of motion control - long duration with low energy consumption and very fast action with large energy consumption is a relevant feature for robotic devices providing efficient capability to react on sudden obstacles or another need for change of the trajectory of different moving parts of such devices.

In order to model excitation activity, we based on Hodgkin-Huxley type equations [2; 3], describing the excitation process in a single cell membrane. Sum of ionic currents flowing across the cell membrane, including external current representing external excitation, is described as follows:

$$
\begin{aligned}
-C d V / d t=m^{3} h g_{N a}\left(V-V_{N a}\right) & +g_{K} n^{4}\left(V-V_{K}\right)+g_{\text {leak }}\left(V-V_{\text {leak }}\right)+I_{\text {ext }}(t) \\
d m / d t & =\alpha_{m}(1-m)-\beta_{m} m \\
d h / d t & =\alpha_{h}(1-h)-\beta_{h} h \\
d n / d t & =\alpha_{n}(1-n)-\beta_{n} n
\end{aligned}
$$

where $C$-capacitance of a cell membrane, $\mu \mathrm{F} \cdot \mathrm{cm}^{-2}$;

$V$ - transmembrane potential, $\mathrm{mV}$;

$g$ - conductivity of sodium (Na), potassium (K) and leakage currents correspondingly, $\mathrm{mS} \cdot \mathrm{cm}^{-2}$;

$V_{N a}, V_{K}$ and $V_{\text {leak }}$ - reversal potential of sodium, potassium and leakage currents correspondingly, $\mathrm{mV}$;

$I_{\text {ext }}(t)$ - external current, imitating external excitation, $\mathrm{mA} \cdot \mathrm{cm}^{-2}$;

$t$ - time, ms;

$m, n-$ activation variables of sodium and potassium currents correspondingly;

$h$ - inactivation variable;

$\alpha$ and $\beta$-activation and inactivation variable coefficients - nonlinear functions of transmembrane potential.

The model allows to imitate the influence of the kinetic properties of ionic currents on mechanical force and to describe the contraction velocity as the function of ionic current properties. HodgkinHuxley model was chosen due to a possibility of physiological validation of equations, and it provides an option to include electro-mechanical coupling into a model, thus linking excitation and mechanical force generation mechanisms. The model was used in order to determine the key parameters, which allow to imitate differences of red and white muscle fibres. Due to experimentally known 
electrophysiological characteristics of ionic currents it will be possible to produce more accurate mathematical models for red and white mucle cells and after to use such models to investigate differences in behaviour of different types of muscle cells in force generation.

\section{Materials and Methods}

We used Mathcad software to resolve the set of Hodgkin-Huxley equations in order to find transmembrane potential $\mathrm{V}$ variations in time. To include differences in the properties of white and red muscular fibres, based on the modelling experiments, we used the data for the fibre cell membrane capacitance $C$ and activation kinetic variable of sodium current as the key parameters affecting differences in performance for both types of fibres. Initial conditions for variables in equations were put in correspondence to excitation of a fibre cell in a state of rest. In order to introduce action of external current representing external excitation, we used the following expression for the current:

$$
\text { Iext }_{1}=k \Phi(i-\text { ton } / s) \Phi(\text { toff } / s-i)
$$

where $k$-current amplitude, duration of external current was introduced by a product of onset and offset functions $\Phi$ depending on the values of onset time "ton" and offset time "toff" and integration parameters such as the integration step number " $i$ ".

As an example, we used duration of external current in amount of $60 \mathrm{~ms}$. When modelling red muscle fibres we used different values of external current with a goal to determine when autooscillatory regime occurs (Fig. 1-3).

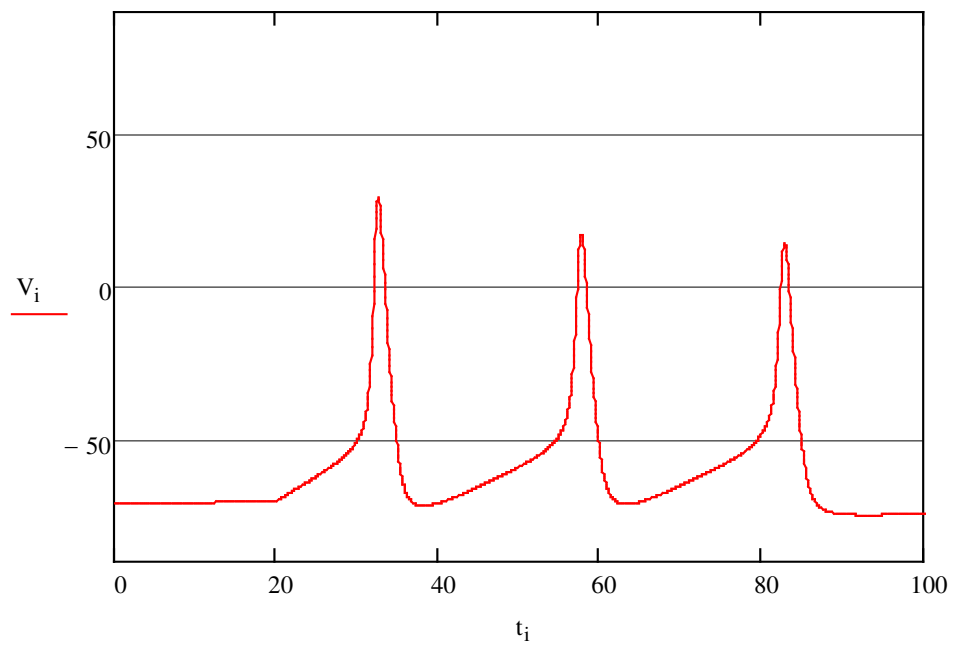

Fig. 1. Dependence of transmembrane potential $V_{i}$ in time $t_{i}, C_{m}=9.5 \mu \mathrm{F} \cdot \mathrm{cm}^{-2}$

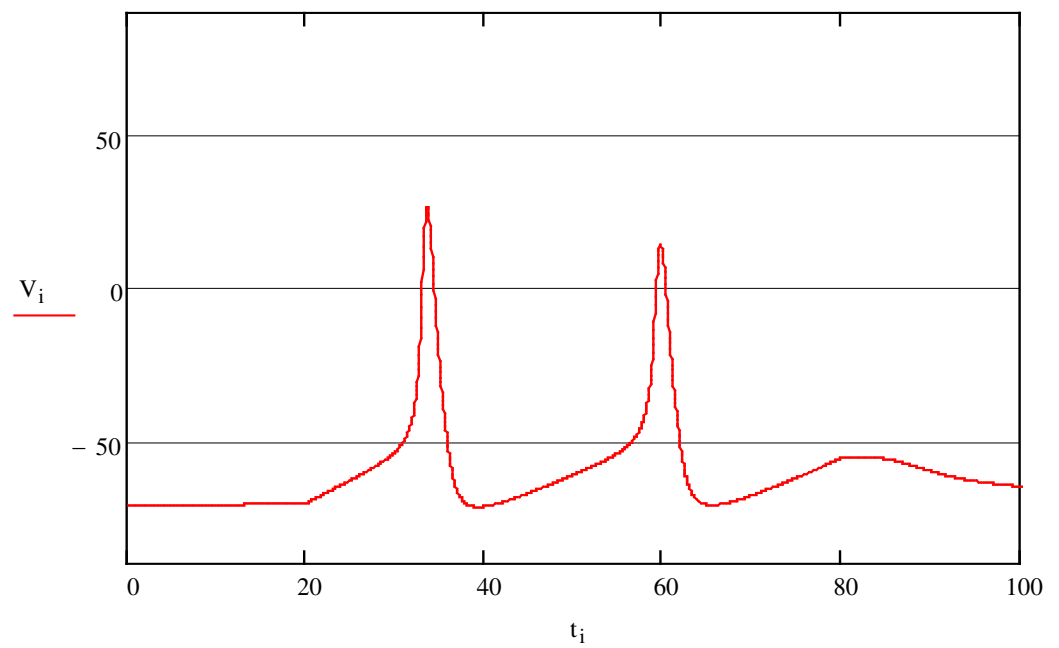

Fig. 2. Dependence of transmembrane potential $V_{i}$ in time $t_{i}, C_{m}=10,7 \mu \mathrm{F} \cdot \mathrm{cm}^{-2}$, increased capacitance leads to lower excitation activity 
As it is shown in Fig. 1 and 2, variation of capacitance from $C=9.5 \mu \mathrm{F} \cdot \mathrm{cm}^{-2}$ to $C=10.7 \mu \mathrm{F} \cdot \mathrm{cm}^{-2}$ causes autooscillations to terminate faster. One can also conclude that the fibre cell membrane capacitance value affects the speed of generation of muscular force, since the excitation impulse decreases both in amplitude and in onset velocity with increased capacitance. It should be noted that in case of a very strong external excitation signal it is possible to model existence of low amplitude depolarization oscillations, which do not lead to the development of mechanical force (Fig. 3).

In case of white muscle fibres we made variations of capacitance in a model, as a result for similar values of external signal the fibre generated action potentials with larger amplitude and larger onset velocity if compared with the red fibre cell model (Fig. 4).

Larger and faster depolarization leads to increased force value due to increased sodium influx through the cell membrane.

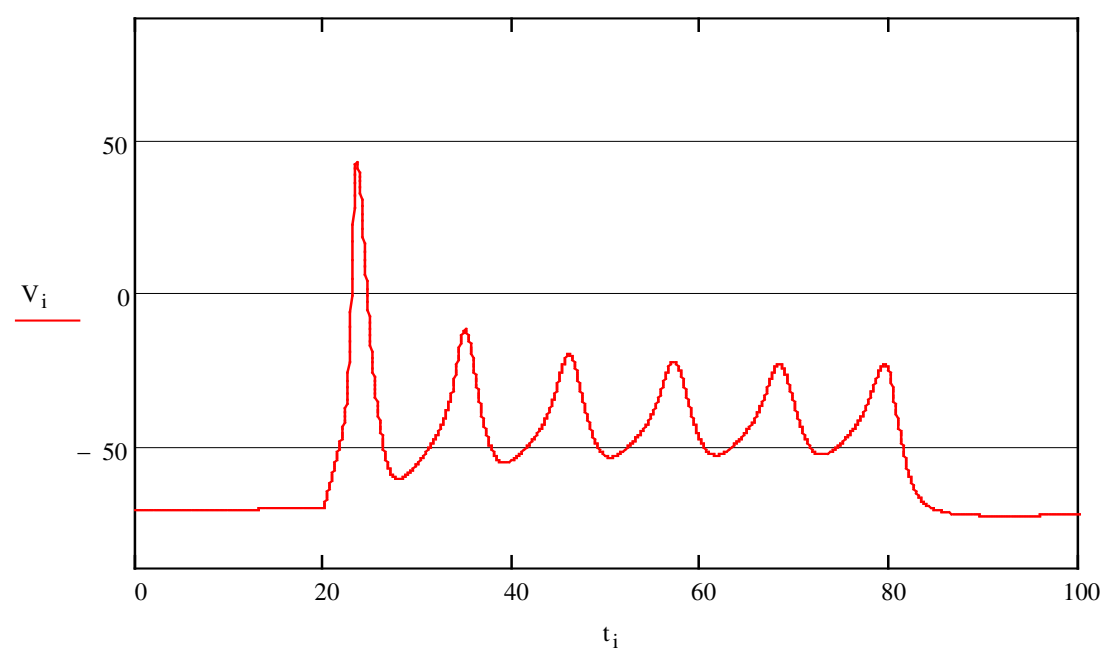

Fig. 3. Strong external signal leads to low amplitude auto-oscillations

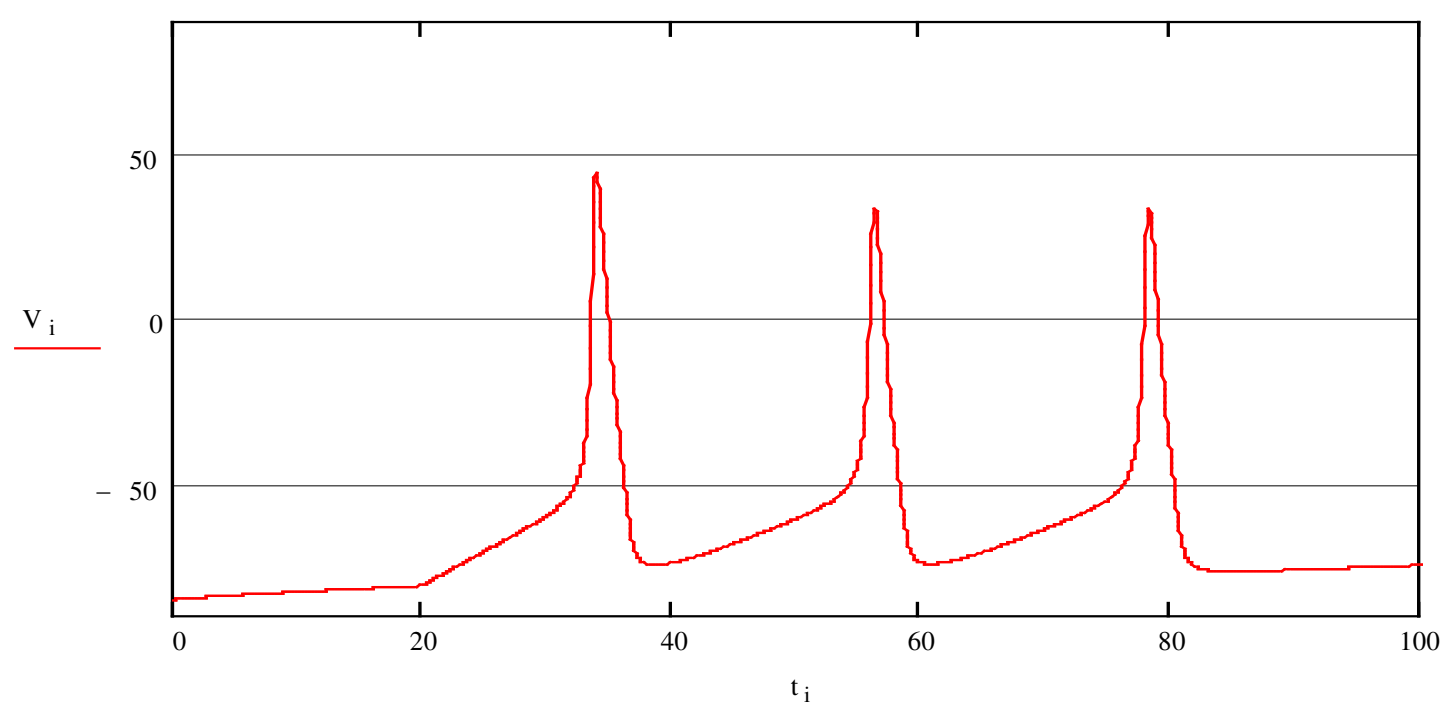

Fig. 4. Transmembrane potential variations in time in case of white muscle fibre model

\section{Results and discussion}

Figures 1-4 show that the use of Hodgkin-Huxley type equations allows to perfom quantitative analysis and to determine differences in the main excitation parameters, affecting force generation in red and white muscles. Two key parameters - the cell membrane capacitance and sodium current activation parameter - allowed us to show different behaviour of white and red muscle fibres. Quantitative characteristics of those parameters are easy to update based on new experimental results. Use of Hodgkin-Huxley type models proved to be an efficient tool to investigate action of calcium blocking drugs to oscillating activity of heart cells [4]. A similar approach based on the experimental 
results can be used also for muscle cell fibres in order to have quantitative evaluation of enhancing or disturbing mechanical performance of different muscle fibres due to drug action. As it is shown in the modelling experiments, it is possible to determine the values of external current when there is no full repolarization in cells and thus force generation is disturbed or not possible at all. Further modelling experiments will be performed to determine critical values of activation parameters leading to decrease of force generation. For that purpose further development of electro-mechanical coupling models will be needed.

The results can be used in design of moving robotic devices, which need to include an option for sudden change of projectile due to various reasons. Further analysis should search for such drives, e.g., gyroscopic drive for a robotic fish [5] - drives which are able to perform that kind of dual action - long duration steady-state motion with low energy consumption and fast change in motion parameters with large energy consumption for a short time interval. The ideas of muscular force development can be implemented in the design and development of robotic devices, e.g., fish robots [6].

\section{Conclusions}

1. Investigation of differences in performance in mechanical force generation by using known Hodgkin-Huxley type models proves to be an efficient tool to determine the main parameters affecting the excitation amplitude and onset velocity.

2. In order to imitate behavior of living moving objects it is essential to include different force generation mechanisms, similar to those in muscles, which allow living objects to react on sudden necessity to change a trajectory - to avoid obstacles or attacks, or to perform attacks, etc.

3. Such dual force generation mechanisms could be a part of moving robotic devices, e.g., by using drives able to perform such dual force generation.

\section{References}

1. Zierath J.R., Hawley J.A. (2004) Skeletal muscle fibre type: influence on contractile and metabolic properties. PLoS Biol 2(10): e348, October 12, 2004. [online][11.12.2016] Available at: https://doi.org/10.1371/journal.pbio.0020348

2. Hodgkin A.L, Huxley A.F. The components of membrane conductance in the giant axon of loligo. Journal of Physiology. No.116, 1952, pp.473-496

3. Bronzino J.D. The biomedical engineering handbook. Hartford, Connecticut: CRC Press, IEEE Press, 1995. pp. 145, 150-151.

4. Plonsey R., Starmer F., Tipans I. The quantitative investigation of the binding process of calcium blocking drugs in sinoatrial node. Journal of Theoretical Biology, 149, 1991, pp.55-61.

5. Viba J., Auzins J., Beresnevich V., Cifanskis S., Kaktabulis I., Kulikovskis G., Tipans I., Melnikovs A., Kruusmaa M., Megill W. Patent LV 14386, Republic of Latvia, Int.Cl. B63 H1/00. Gyroscopic method for forming motive force of floating vehicle. Patenti un preču zīmes, 2011, No.12, p.1774.

6. Tipans I., Cifanskis S., Viba J., Jakushevich V., Grasmanis B., Kulikovskis G.. Synthesis of a robot fish prototoype, Engineering for Rural Development Proceedings of the 10th International Scientific Conference, Volume 10, 2011, pp. 458-463. 